\title{
Pituitary lesions in breast cancer patients: A report of three cases
}

\author{
NICOLETTA FORTUNATI $^{1-3}$, FRANCESCO FELICETTI ${ }^{1,3,4}$, MICHELA DONADIO $^{2}$, EMIDIO GROSSI $^{3}$, \\ FEDERICA MICHELON $^{3}$, GIULIANA RITORTO ${ }^{2}$, EMANUELA ARVAT $^{3,4}$ and ENRICO BRIGNARDELLO ${ }^{1}$ \\ ${ }^{1}$ Transition Unit for Childhood Cancer Survivors; ${ }^{2}$ Breast Unit; ${ }^{3}$ Oncological Endocrinology Unit, \\ Department of Oncology, Città della Salute e della Scienza di Torino, Turin 10126; ${ }^{4}$ Department of Medical Sciences, \\ University of Torino, Turin 10126, Italy
}

Received March 13, 2014; Accepted December 12, 2014

DOI: $10.3892 / 01.2015 .3149$

\begin{abstract}
Pituitary metastases occur in 6-8\% of breast cancer cases, but are seldom diagnosed and rarely reported. Therefore, it can be challenging to establish a clinical differential diagnosis, and at present, a definitive criteria is not available. The present study discusses the pituitary lesions identified in three patients with breast cancer, and describes their management within the collaborative framework of the Breast Unit at the Città della Salute Hospital, which also included assessment by endocrinologists. The patients were evaluated for anterior and posterior pituitary function, the appearance of the pituitary upon magnetic resonance imaging (MRI), and the oncology status and treatment. In addition, successive analysis of prolactin levels and the MRI was performed. The patients, aged 75,83 and 76 years old, differed in their clinical presentation and successive evolution. One patient demonstrated an abrupt onset of diabetes insipidus, the second exhibited overt hypopituitarism and the final patient had a pituitary mass discovered by chance. Cases one and three exhibited systemic spread of the breast cancer, with bone and/or parenchymal metastasis, but not brain metastasis. Case two presented with a secondary pituitary tumour alone. In case three, a secondary nature to the pituitary lesion was unlikely, since there was no lesion evolution evident following MRI and as stable prolactin levels were observed over the course of the study period. By contrast, case one presented with a rapid increase of sellar lesions on MRI, together with a progressive rise in prolactin levels. Taking into account the frailty of breast cancer patients who are monitored for disease progression, management in a collaborative framework, such as at the Breast Unit, makes it possible to establish a diagnosis of sellar lesions, which is adequate for the comprehensive management of the patient
\end{abstract}

Correspondence to: Dr Nicoletta Fortunati, Oncological Endocrinology Unit, Department of Oncology, Città della Salute e della Scienza di Torino, 3 Via Genova, Turin 10126, Italy

E-mail: nfortunati@cittadellasalute.to.it

Key words: breast neoplasm, pituitary diseases, metastases with successive pituitary MRIs and prolactin evaluations, and avoids unnecessary invasive neurosurgery.

\section{Introduction}

In breast cancer patients, the development of metastatic disease is considered to be the major cause of mortality (1). The invasion-metastasis cascade is a multistep process in which breast cancer cells invade the systemic circulation and distant tissues, and cause the metastatic colonization of different organs, including the bones, lungs and central nervous system (2). Being highly vascularized, the pituitary gland is a target structure for metastatic spread, accounting for $0.14-28 \%$ of all reported brain metastases following autopsy (3). In total, 6-8\% of breast cancer cases demonstrate metastatic spread to the pituitary gland (4). Despite this, pituitary metastases are seldom diagnosed and rarely described in surgical series, overall representing just $1 \%$ of surgically-resected tumours in the sellar region (5). The clinical differential diagnosis of benign lesions in the sellar region is challenging, and at present, a definitive clinical criteria is not available (6). The majority of patients may be asymptomatic, but the most common symptoms include central diabetes insipidus, anterior hypopituitarism, visual loss and central nervous system disorders (7). Furthermore, the treatment of secondary pituitary lesions, for example with surgery, radiotherapy or chemotherapy, is far from being defined.

In the Città della Salute Hospital (Turin, Italy), breast cancer patients are referred to the Breast Unit, where surgeons, oncologists and other specialists have established a collaborative framework. Recently, endocrinologists have also been included in the staff of the Breast Unit, from 75 patients referred to the endocrinologists between April 2012 to January 2014, three cases of sellar region lesions have been identified. The present study describes three cases of pituitary lesions in breast cancer patients who demonstrated different clinical presentations and histories, and discusses the correct diagnostic process and management. Patients provided written informed consent.

\section{Case report}

Case one. A 75-year-old female presented at the Breast Unit of the Città della Salute Hospital in September 2012 and was diagnosed with infiltrating ductal breast cancer [pathological 
staging, PT1c pNx; oestrogen receptor (ER)- and progesterone receptor-negative; Her2/neu, 80\%) 12 years previously. Following left quadrectomy, the patient received chemotherapy (6 cycles, each cycle lasts 21 days) consisting of fluorouracil $\left(600 \mathrm{mg} / \mathrm{m}^{2}\right.$ on day 1$)$, epirubicin $\left(100 \mathrm{mg} / \mathrm{m}^{2}\right.$ on day 1$)$ and cyclophosphamide $\left(600 \mathrm{mg} / \mathrm{m}^{2}\right.$ on day 1$)$, and underwent radiotherapy on the other breast. One year later, the patient presented with secondary axillary node lesions ( $\mathrm{pN} 2$, node $6 / 11)$ and received chemotherapy with taxotere $\left(100 \mathrm{mg} / \mathrm{m}^{2}\right.$ on days $1-14,8$ cycles, each cycle lasting 21 days). Subsequently, a further local recurrence, with pleural effusion, was identified, which required chemotherapy (8 cycles, each lasting 21 days) with vinorelbine (35 mg/m $\mathrm{m}^{2}$ on days $\left.1-8\right)$ and capecitabine $\left(1,250 \mathrm{mg} / \mathrm{m}^{2}\right.$ twice daily, on days $\left.1-14\right)$, which was administered systemically. Following this, the patient began treatment with examestane $(25 \mathrm{mg} /$ day). Despite therapy, the disease progressed with bone dissemination, which was treated with zolendronate. At the end of 2011, the patient complained of polydipsia, with a water intake of $\sim 3$ litres in $24 \mathrm{~h}$, and polyuria, particularly at night, with diuresis of $\sim 5$ litres every $24 \mathrm{~h}$. The laboratory data revealed a low urine density (normal, $1005-1028 \mathrm{~g} / \mathrm{cm}^{3}$ ), a high serum sodium concentration (147 mmol/l; normal, 135-145 mmol/l) and a slight increase in prolactin levels $(30.4 \mathrm{ng} / \mathrm{ml})$. Normal ranges are shown in Table I. The fasting blood glucose (normal, 70-109 mg/dl) and anterior pituitary hormone levels were in the normal range. MRI revealed an increase in the size of the pineal and pituitary glands, which was characterized by a heterogeneous T2 signal and by contrast enhancement. The pituitary stalk was enlarged and demonstrated strong enhancement when using gadolinium contrast medium. The patient was treated with $60 \mathrm{mg} /$ day oral desmopressin, and demonstrated a good initial response, with the serum sodium level decreasing to $141 \mathrm{mmol} / \mathrm{l}$ and diuresis to $\sim 50 \%$. After three months, the prolactin levels were significantly elevated and reached $141 \mathrm{ng} / \mathrm{ml}$, without any further worsening of the diuresis pattern or any visual defects. The MRI scan was repeated and revealed a clear progression of the pineal-pituitary lesion, with an increased involvement of the stalk and optic chiasms. The patient was considered eligible for stereotactic radiotherapy (STR) of the sellar region, however, shortly after, the general and respiratory condition deteriorated and the patient succumbed to the disease.

The hormone levels and MRI results are presented in Table I and Fig. 1A, respectively.

Case two. An 83-year-old female was admitted to the Emergency Department of the Città della Salute Hospital in early 2012 with symptoms of weakness, weight loss, hypotension and recurrent dizziness. A physical examination revealed the presence of a palpable, hard and irregular mass in the right breast. The patient had not sought any previous medical advice, but reported that the mass had been apparent for approximately two years. Following pituitary function evaluation, reduced levels of morning serum cortisol, free-urinary cortisol, adrenocorticotropic hormone, free triiodothyronine (fT3), free thyroxine (fT4), thyroid-stimulating hormone (TSH), luteinizing hormone (LH) and follicle-stimulating hormone (FSH) were observed. By contrast, prolactin serum levels were increased (152 ng/dl). Following the start of hormone replacement therapy with hydrocortisone
(20 $\mathrm{mg} /$ day) and L-thyroxin (75 $\mu \mathrm{g} / \mathrm{day})$, the patient underwent a pituitary MRI, which revealed a large lesion with sovra-sellar extension, optic chiasm infiltration and high post-gadolinium enhancement. The visual field examination revealed the presence of a temporal right superior quadrantanopsia, despite the absence of overt visual disturbances. Therefore, the patient underwent trans-sphenoidal surgery. The pituitary lesion was removed and upon histological examination, was diagnosed as a metastasis of mucinous breast cancer. A ultrasound-guided percutaneous fine-needle aspiration biopsy was performed upon the breast node, which established a positive result for infiltrating low-grade carcinoma with mucinous characteristics. No secondary lesions, other than the pituitary metastasis, were observed. No breast surgery was performed, however, treatment with anastrazole (1 mg/day) was initiated. The one-year follow-up examination revealed no evidence of disease progression. The patient was subsequently lost to follow-up.

The hormone levels and MRI results are presented in Table I and Fig 1B, respectively.

Case three. A 76-year-old female was referred to the Breast Unit of the Città della Salute Hospital in April 2012 following evidence of a sellar lesion with a polylobulate aspect, measuring $20 \mathrm{~mm}$, upon brain MRI. The mass demonstrated high signal intensity upon T1-weighted images, (suggesting lipid presence), and low signal intensity upon T2-weighted images, with sovra-sellar extension and pituitary involvement.

In 1997, the patient was diagnosed with breast cancer and underwent breast conservative treatment, consisting of a quadrantectomy and radiotherapy of the right breast. The histological examination established a diagnosis of ER- and cErb2-positive ductal infiltrating breast cancer. The patient subsequently received adjuvant chemotherapy [epirubicin (120 mg/m² , once a week for 3 cycles)] and radiotherapy to the other breast. Evidence of recurrence was not observed until 2004, when the patient underwent chemotherapy [cyclophosphamide (100 mg, twice a day, on days 1-14), methotrexate $\left(40 \mathrm{mg} / \mathrm{m}^{2}\right.$ on days $\left.1-8\right)$ and fluorouracil (600 mg/m $\mathrm{m}^{2}$ on days $\left.1-8\right)$, every 28 days, total of 6 cycles] and surgery, which consisted of a right mastectomy and a lymphadenectomy for the removal of nodal metastases in the right axilla. Subsequently, treatment with anastrazole (1 mg/day) was initiated. Disease progression was not observed until January 2010, when liver and bone metastases were identified. The patient was therefore treated with chemotherapy (3 cycles, each lasting 21 days), consisting of vinorelbine $\left(35 \mathrm{mg} / \mathrm{m}^{2}\right.$ on days $1-8)$ and capecitabine $\left(1,250 \mathrm{mg} / \mathrm{m}^{2}\right.$, twice a day on days 1-14), which lead to regression of the liver and bone lesions. In early 2011, a brain CT scan and MRI revealed only the presence of the aforementioned pituitary lesion. The pituitary function results revealed low levels of fT3, fT4 and TSH, extremely low levels of FSH and $\mathrm{LH}$ and a moderate increase in the level of prolactin $(72 \mathrm{ng} / \mathrm{ml})$. The adrenal function was normal, and no clinical or laboratory findings suggestive of diabetes insipidus were revealed. Hormone replacement therapy with L-thyroxine (100 $\mu \mathrm{g} /$ day) was administered. Alterations in the visual field were not observed.

An MRI scan of the pituitary region repeated six months later did not reveal a significant difference in the mass 
Table I. Hormone assays at the time of pituitary mass diagnosis.

\begin{tabular}{|c|c|c|c|c|}
\hline Hormone assay & Case one & Case two & Case three & Normal \\
\hline ACTH (pg/ml) & 12.0 & 8.0 & 36.0 & $<46.0$ \\
\hline Morning cortisol $(\mu \mathrm{g} / \mathrm{dl})$ & 20.7 & 1.0 & 10.2 & $6.2-19.4$ \\
\hline Prolactin (ng/ml) & 35.5 & 152.5 & 72.8 & $4.8-23.3$ \\
\hline $\mathrm{TSH}(\mu \mathrm{IU} / \mathrm{ml})$ & 2.2 & 2.0 & 0.8 & $0.37-4.2$ \\
\hline fT4 $(\mathrm{pg} / \mathrm{ml})$ & 11.5 & 5.4 & 7.0 & $9.3-17.0$ \\
\hline fT3 (pg/ml) & 3.2 & 1.4 & 3.3 & $2.6-4.4$ \\
\hline FSH (mIU/ml) & 88.4 & 0.5 & 6.3 & a \\
\hline LH (mIU/ml) & 38.0 & $<0.1$ & 1.2 & $\mathrm{~b}$ \\
\hline IGF1 (ng/ml) & 121.0 & 59.0 & 72.7 & $110-385$ \\
\hline p-Osmolality (mOsm/kg) & 294.0 & 297.0 & 290.0 & \\
\hline u-Osmolality (mOsm/kg) & 156.0 & 622.0 & 562.0 & \\
\hline
\end{tabular}

${ }^{a}$ Normal range: FSH (follicular phase), 3.5-12.5 mUI/l; FSH (luteal phase), 1.7-17.7 mUI/l; FSH (menopause), 5.8-134.8 mUI/l. ${ }^{\text {DNormal }}$ range: LH (follicular phase), 2.4-12.6 mUI/l; LH (luteal phase), 1.0-11.4 mUI/l; LH (menopause), 7.7-58.5 mUI/1. ACTH, adrenocorticotropic hormone; TSH, thyroid-stimulating hormones; fT4, free thryoxine; fT3, free triiodothyronine; FSH, follicle-stimulating hormone; LH, luteinizing hormone; IGF1, insulin-like growth factor-1; p, plasma; $u$, urine.

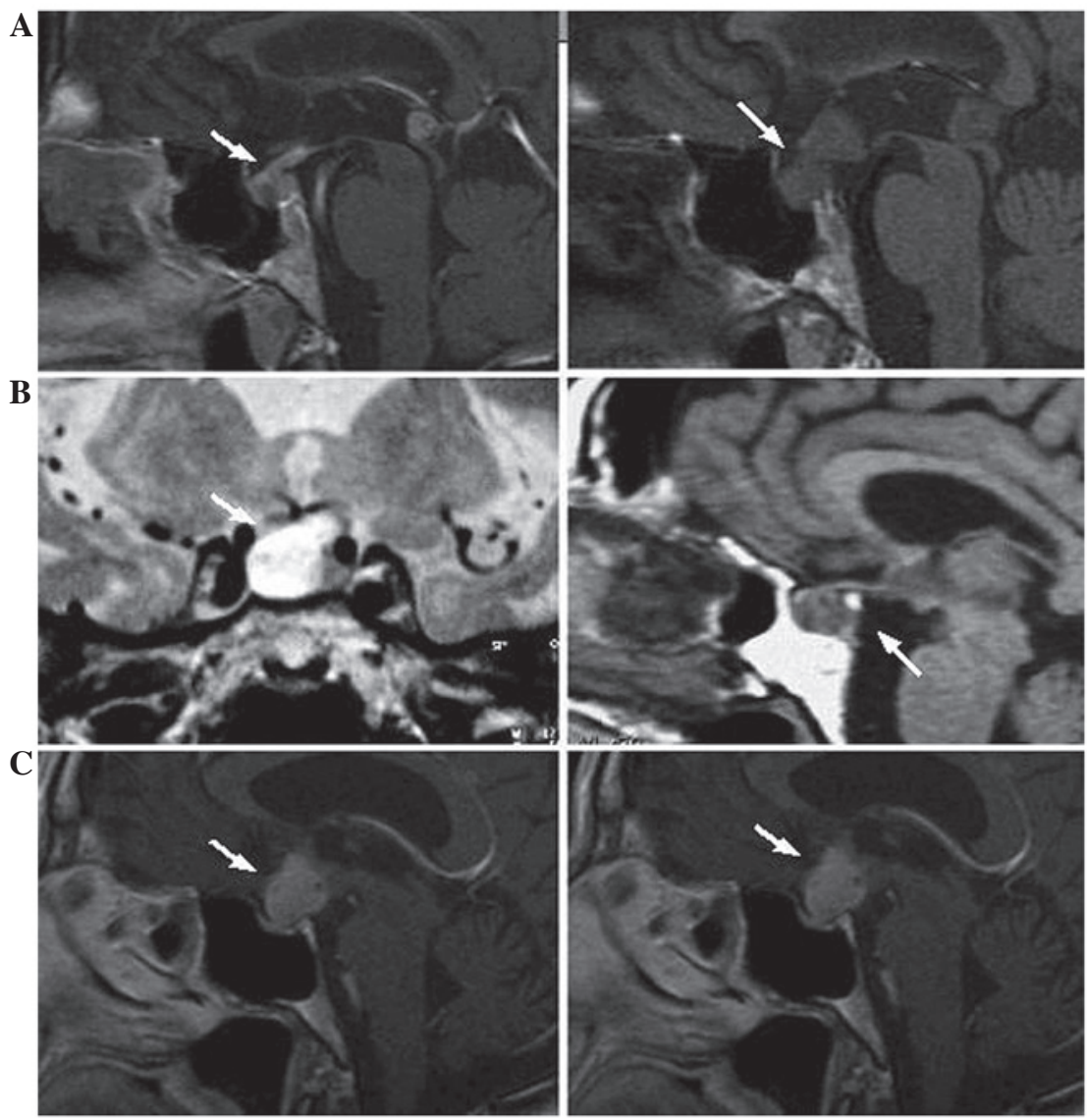

Figure 1. Magnetic resonance imaging (MRI) revealing the pituitary lesions. (A) MRI from case one. Left, at the time of diagnosis, and right, six months later. (B) MRI from case two. Left and right images taken prior to the trans-sphenoidal surgery. (C) MRI from case three. Left, at the time of diagnosis, and right, six months later. Arrows indicate lesion sites.

characteristics. The prolactin levels were also monitored every three months, and demonstrated no significant modifications with respect to the basal level. The clinical and radiological features of the pituitary mass were suggestive of a craniopharyngioma, however, taking into account the oncology history and prognosis, and also the stability of the neurological symptoms, surgical treatment was excluded. The MRI results and prolactin levels remained stable one year after 
the diagnosis of the pituitary lesion. While the pituitary mass did not increase in size, further progression of the secondary liver lesions was observed, and therefore the patient continues to receive chemotherapy.

The hormone levels and MRI are presented in Table I and in Fig. 1C, respectively.

\section{Discussion}

Since the first reported case of a pituitary metastasis by Benjamin (8) in 1857, the number of recorded cases has increased over time. At present, it is reported that following autopsy, pituitary lesions account for $0.14-28.1 \%$ of all brain metastases $(3,6,9)$. Despite this, clinical detection and management of the lesions remain complex and ambiguous.

The current literature regarding pituitary metastases generally describes lesions discovered by neurosurgeons or endocrinologists. The present study reports an integrated management of breast cancer patients at the Breast Unit at the Città della Salute Hospital, where oncologists and endocrinologist work together. The present study therefore aimed to delineate how to diagnose a breast cancer patient when the suspicion of a pituitary lesion arises. Between April 2012 and January 2014, 75 breast cancer patients, who had been referred to the Endocrinology Service of the Breast Unit, were considered for use in the present study. Of these, three patients, who represented $4 \%$ of the total population, presented with a pituitary mass.

It is recognized that pituitary metastasis usually affects elderly patients in the sixth or seventh decades of life (10). Furthermore, pituitary metastases, which are more often observed in breast and lung cancer patients, are usually part of a systemic spread, and are frequently associated with bone localization (7). The patients included in the present study were older than 70 years, which suggests that age is an important factor to be considered. With regard to pituitary lesions and their association with disease progression and systemic spread, in the present study, it was true only in case one. In case two, the pituitary metastasis was the first and only sign of breast cancer spread, and in case three, where the exact nature of the lesions was unknown (craniopharyngioma was clinically diagnosed), the breast cancer progression was observed later without any modification of the pituitary lesion.

The clinical presentation of a sellar lesion is believed to be suggestive of a pituitary metastasis when it is characterized by the abrupt appearance of diabetes insipidus $(3,7)$. This presentation has been associated with a poor prognosis within one year of a diabetes insipidus diagnosis. Metastatic spread is more common to the pituitary posterior lobe, mainly due to the particular anatomy of the supplying arteries. While the anterior lobe lacks a direct arterial blood supply, the posterior lobe is directly supplied by the hypophyseal arteries (7). Therefore, the involvement of the anterior lobe is believed to be a result of contiguous spread from the posterior lobe. Previously, it was reported that diabetes insipidus occurred in $70 \%$ of symptomatic cases of pituitary metastasis, and that anterior pituitary deficiency was identified in $15 \%$ of those patients (7). In the present study, pituitary metastasis was diagnosed in case one following symptoms of diabetes insipidus, and was subsequently associated with a poor prognosis. In case three,

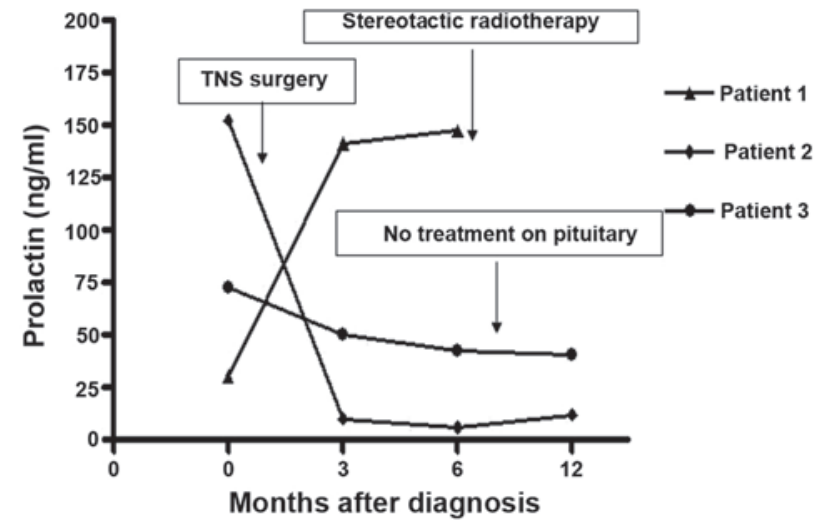

Figure 2. Prolactin levels evaluated in the three patients at the time of pituitary lesion diagnosis, and at three and six months later. TNS, trans-sphenoidal.

where the pituitary lesion was found not to be malignant, an anterior pituitary partial deficiency, which was limited to thyroid function, was observed. Case two presented with overt hypopituitarism and a well-demonstrated deficiency in adrenal and thyroid function.

The symptoms of diabetes insipidus in a patient with metastatic breast cancer may suggest the presence of a pituitary metastasis (11), and therefore should prompt a pituitary MRI scan. However, it should be noted that the absence of diabetes insipidus should not completely dismiss the potential occurrence of hypophyseal spread.

The patients in the present study did not present with visual disturbances, headaches or ophthalmoplegia, however, this was not of much use during the differential diagnosis, as such symptoms are frequently observed in patients with pituitary adenomas (11).

The final endocrine aspect to be discussed is the level of prolactin at diagnosis and its behaviour. A previous study reported that hyperprolactinaemia is present in $6.3 \%$ of pituitary metastases, and that levels $>200 \mathrm{ng} / \mathrm{ml}$ are suggestive of a prolactinoma (7). Mean prolactin levels in cases of pituitary metastasis are $\sim 150 \mathrm{ng} / \mathrm{ml}$ and are believed to result from stalk compression (12). In agreement with this, none of the patients in the present study demonstrated prolactin levels $>200 \mathrm{ng} / \mathrm{ml}$, but the two with pituitary metastases did exhibit high prolactin levels of $\sim 150 \mathrm{ng} / \mathrm{ml}$ on at least one occasion during the study. The opportunity to follow-up patients during the months following the diagnoses allowed for notable observations to be made (Fig. 2). The change in prolactin levels over time could be suggestive of the evolution of the lesion. Only case three presented with moderately elevated prolactin levels, but these remained stable over time. In the other patients, prolactin was $>100 \mathrm{ng} / \mathrm{ml}$ at diagnosis, or increased above this value as the lesion progressed. Therefore, this last observation should be taken into consideration. When possible, prolactin levels should be monitored over time, with increases suggesting the potential presence of an evolving pituitary mass. This was confirmed in case one by MRI, which demonstrated a clear increase in the size of the pituitary lesion, and the presence of stalk involvement over time. In case three, the lesion remained stable on MRI, as did the prolactin levels, despite breast disease progression. Furthermore, since none of the patients 
took anti-dopaminergic agents during the endocrinological assessment, the prolactin levels have to be considered as a reliable hormonal marker. Therefore, it can be suggested that in breast cancer patients with a suspected pituitary metastasis, an evaluation of prolactin levels and an MRI scan at different time intervals may aid in the differential diagnosis.

The only approach able to provide a definite diagnosis is a biopsy of the sellar lesion, which requires trans-sphenoidal surgery performed by an experienced neurosurgical team. Previously, it has been reported that surgery, despite providing pathological confirmation of the disease, does not increase the mean survival time $(5,6)$ of 6-7 months $(9)$, but does improve quality of life. Therefore, surgery should be performed in patients who have experienced a consistent reduction in the quality of their life (5) or in order to decompress the optic nerves and chiasm (13). A surgical approach to obtain a histological diagnosis is advisable when the primary site of the tumour is unknown. At the Breast Unit, MRI and prolactin evaluation at different time-points (e.g., intervals of one to three months), together with a pituitary functional assessment at the first observation, are currently performed and should provide a clinician with the correct interpretation of the biological behaviour of the sellar lesion. Finally, an exhaustive evaluation of patient quality of life, life expectancy and surgical risk has to be performed in each case in order to make an informed decision regarding surgery.

As suggested by previous studies, when overt symptoms, such as visual disturbances and headaches, or a marked increase in the size of the lesion over a short period of time requires treatment, but surgery cannot be considered, an attractive and effective therapeutic option is radiation therapy, either traditional or sterotactic $(5,14)$. STR was suggested for one of the patients in the present study, but disease spread with critical clinical deterioration did not allow for any further treatment.

Despite no published literature existing on the potential molecular pathways involved in breast cancer cell spread to the pituitary gland, certain putative candidates appear noteworthy, particularly pituitary tumour-transforming gene 1 (PTTG1) (15). PTTG1 is a pituitary-derived transforming gene, and its cognate protein, PTTG1, exhibits a high-grade homology to securin, which in vertebrates regulates sister chromatid separation during mitosis (15). Furthermore, PTTG1 has been reported to be overexpressed in several hormone-related tumours, such as pituitary, thyroid, breast, ovarian and uterine cancers. In addition, PTTG1 is associated with tumour metastasis and its expression in breast cancer has been proposed to be a marker of aggressiveness. Despite being only speculative, PTTG1 may be involved in the spread of breast cancer to the pituitary, and therefore requires future investigation.

In conclusion, the present study reported three cases of breast cancer with corresponding pituitary masses. Of the three cases, one behaved as a benign lesion and demonstrated characteristics of a craniopharyngioma, while the other two were secondary lesions of the primitive neoplasm. It is suggested that when breast cancer patients are managed within a collaborative framework, such as the Breast Unit at the Città della Salute Hospital, it is possible to establish an adequate diagnosis of sellar lesions and avoid unnecessary invasive neurosurgery, which is favourable when considering the frailty of these patients. Repeated pituitary MRI and prolactin evaluation following a correct pituitary function study may be useful tools for making a therapeutic decision. Finally, the present study suggests a valuable role for endocrinologists during the diagnosis and treatment of breast cancer patients.

\section{Acknowledgements}

The authors would like to thank Dr Dino Daniele and Dr Mauro Bergui (Neuroradiology Unit) for providing assistance with the MRI scans.

\section{References}

1. Gupta GP and Massagué J: Cancer metastasis: building a framework. Cell 127: 679-695, 2006.

2. Nguyen DX, Bos PD and Massagué J: Metastasis: from dissemination to organ-specific colonization. Nat Rev Cancer 9: 274-284, 2009.

3. Sioutos P, Yen V and Arbit E: Pituitary gland metastases. Ann Surg Oncol 3: 94-99, 1996.

4. Fassett DR and Couldwell WT: Metastases to the pituitary gland. Neurosurg Focus 16: E8, 2004.

5. Zoli M, Mazzatenta D, Faustini-Fustini M, Pasquini E and Frank G: Pituitary metastases: role of surgery. World Neurosurg 79: 327-330, 2013

6. Marsh JC, Garg S, Wendt JA, Gielda BT, Turian JV and Herskovic AM: Intracranial metastatic disease rarely involves the pituitary: retrospective analysis of 935 metastases in 155 patients and review of the literature. Pituitary 13: 260-265, 2010.

7. Komninos J, Vlassopoulou V, Protopapa D, Korfias S, Kontogeorgos G, Sakas DE and Thalassinos NC: Tumors metastatic to the pituitary gland: case report and literature review. J Clin Endocrinol Metab 89: 574-80, 2004.

8. Benjamin L: Ein Krebsfall. Virchows Arch Path Anat 12: 566-569, 1857

9. Chiang MF, Brock M and Patt S: Pituitary metastasis. Neurochirurgia (Stuttg) 33: 127-131, 1990.

10. Freda PU and Post KD: Differential diagnosis of sellar masses. Endocrinol Metab Clin North Am 28: 81-117, 1999.

11. Shubinger O and Haller D: Metastases to the pituitary - hypothalamic axis. An MR study of 7 symptomatic patients. Neuroradiology 34: 131-134, 1992.

12. Leramo OB, Booth JD, Zinman B, Bergeron C, Sima AA and Morley TP: Hyperprolactinemia, hypopituitarism, and chiasmal compression due to carcinoma metastatic to the pituitary. Neurosurgery 8: 477-480, 1981.

13. Post KD: Pituitary metastases: what is the role of surgery? World Neurosurg 79: 251-252, 2013.

14. Turaka A, Parsons RB and Buyyounouski MK: Radiation therapy for pituitary metastasis: report of four cases. Tumori 98: e1-e6, 2012.

15. Vlotides G, Eigler T and Melmed S: Pituitary tumor-transforming gene: physiology and implications for tumorigenesis. Endocr Rev 28: 165-186, 2007. 